\title{
Phase separation behaviour in blends of isotactic polypropylene and ethylene- propylene diene terpolymer
}

\begin{abstract}
Miscibility and phase separation behaviour in blends of isotactic polypropylene (iPP) and ethylene-propylene diene terpolymer (EPDM, ethylene content 70\%) have been examined by means of depolarized light scattering, polarizing optical microscopy, differential scanning calorimetry and dynamic mechanical methods. A cloud point phase diagram was established by means of light scattering after eliminating the influence of iPP crystal melting on liquidliquid phase separation of iPP/EPDM blends. A lower critical solution temperature (LCST) was observed above the crystallization temperature, but below the melting temperature of iPP. This LCST is thermally reversible, so long as crystallization does not occur in its vicinity. The coupling between the crystal melting and liquid-liquid phase separation has been examined. Crystallization-induced phase separation and morphology development during crystallization of iPP have been investigated.
\end{abstract}

Keyword: Crystallization; iPP/EPDM blends; LCST; Phase separation; Spinodal decomposition 